\title{
O CONTROLE DA TUBERCULOSE NA PERSPECTIVA DA VIGILÂNCIA DA SAÚDE
}

\author{
Tubercul osis in the perspective of Health Surveillance \\ El control de la Tuberculosisen la Vigilancia de Salud
}

\author{
Paula Hino \\ Claudia Benedita dos Santos ${ }^{2}$ \\ Maria Rita Bertolozzi \\ Renata Ferreira Takahashi ${ }^{5}$
}

Tereza Cristina Scatena Villa ${ }^{3}$

\section{RESUMO}

Considerando que o referencial teórico da Vigilância da Saúde (VS) pode constituir um importante modelo para monitoramento da situação de saúde, este estudo reflexivo tem por objetivo discutir as possibilidades de contribuição no controle da Tuberculose. Com base em estudos anteriores de grande escala, a discussão aponta que a identificação de áreas heterogêneas de risco pode fornecer subsídios para a escolha das intervenções direcionadas para as necessidades de grupos mais vulneráveis, contribuindo, assim, para diminuir as iniquidades em saúde. A VS utilizada na Estratégia de Saúde da Família torna-se um profícuo meio de transformar os processos de trabalho em saúde, ao propiciar a compreensão ampliada do objeto da atenção e estimular intervenções nos determinantes da Tuberculose.

Palavras-chave: Tuberculose. Epidemiologia. Saúde Pública. Doenças endêmicas

\begin{abstract}
Health Surveillance (HS) framework is important to become a model best follow up health situation in a community. This reflective study aims to discuss the possibilities of contribution of this framework to tuberculosis control in Brazil. Previous studies in this theme allow confirming that HS is essential to identify heterogeneous area of risk to tuberculosis and also allow to do better interventions according to health needs of social vulnerable groups. Thus is possible to contribute to decrease social inequalities. If the Health Surveillance is used in the Strategy of Family Health, it becomes a useful means to transform the health work process, to provide a broadened understanding of the object of attention and stimulate action on the determinants of tuberculosis.
\end{abstract}

Keywords: Tuberculosis. Epidemiology. Public Health. Endemic Diseases

\section{Resumen}

Considerando que la Vigilancia de Salud (VS) puede constituirse en un importante modelo para monitorizar la situación de la salud, este estudio reflexivo tiene objetivo discutir las posibilidades de contribución de este referencial en el control de la tuberculosis. Apoyados en los estudios anteriores de gran escala, la discusión apunta que la identificación de las áreas heterogéneas del riesgo pueden ofrecer subsidios para la escoja de las intervenciones direccionadas para las necesidades de salud de los grupos más vulnerables, de esa forma contribuyendo para disminuir las iniquidades en salud. El referencial de VS se utiliza en la Estrategia de Salud de la Familia y se convierte en un medio útil para la transformación de los procesos de trabajo en salud, para proporcionar una mayor comprensión del objeto de la atención y estimular la acción sobre los determinantes de la tuberculosis.

Palabras chave: Tuberculosis. Epidemiología. Salud Pública. Enfermedades endémicas.

\footnotetext{
${ }^{1}$ Pós-doutoranda do Departamento de Enfermagem em Saúde Coletiva - Escola de Enfermagem da Universidade de São Paulo. Bolsista CNPq. São Paulo - SP. Brasil.Email: paulahino@yahoo.com.br, ${ }^{2}$ Estatística. Professora Doutora do Departamento de Enfermagem Materno-Infantil e Saúde Pública Escola de Enfermagem de Ribeirão Preto da Universidade de São Paulo. Ribeirão Preto-SP. Brasil. Email: cbsantos@eerp.usp.br, ${ }^{3}$ Enfermeira. Professora Titular do Departamento de Enfermagem Materno-Infantil e Saúde Pública - Escola de Enfermagem de Ribeirão Preto da Universidade de São Paulo. Ribeirão Preto - SP. Brasil. Email: tite@eerp.usp.br, ${ }^{4}$ Enfermeira. Professora Associada do Departamento de Enfermagem em Saúde Coletiva - Escola de Enfermagem da Universidade de São Paulo. São Paulo - SP. Brasil. Email: mrbertol@ysp.br, ${ }^{5}$ Enfermeira. Professora Associada do Departamento de Enfermagem em Saúde Coletiva - Escola de Enfermagem da Universidade de São Paulo. São Paulo-SP. Brasil. Email: rftakaha@usp.br.
} 


\section{INTRODUÇÃO: O CENÁRIO DA TUBERCULOSE}

A Tuberculose $(\mathrm{Tb})$ é uma enfermidade prevenível, curável e considerada prioridade de saúde pública, denominada "calamidade negligenciada". Em estudo realizado com coordenadores do Programa de Controle da Tuberculose do Estado de São Paulo, identificou-se que estes consideram a Tb uma doença "marginalizada", desvalorizada e com pouca visibilidade política, por acometer, na maioria das vezes, grupos populacionais que vivem em condições de pobreza ou miséria e, portanto, com menor vocalização política e capacidade reivindicatória.'

A Organização Mundial da Saúde (OMS) estima que um terço da população mundial, correspondendo a aproximadamente dois bilhões de pessoas, encontra-se infectada pelo Mycobacterium tuberculosis e, dentre eles, oito a nove milhões desenvolvem a doença. Anualmente, cerca de dois milhões de pessoas falecem dessa enfermidade, principalmente em países e regiões com condições socioeconômicas precárias. ${ }^{2}$

0 Brasil ocupa a $14^{\circ}$ posição entre os 22 países responsáveis por $80 \%$ do total de casos de Tb no mundo. Dados da OMS apontam que, no ano de 2007, o Coeficiente de Incidência ( $\mathrm{Cl}$ ) foi de 48 casos/100.000 habitantes e 0 percentual de coinfecção Tb/HIV foi $14 \% .{ }^{3}$ Em relação ao resultado de tratamento, $72 \%$ dos doentes evoluíram para cura, $4,2 \%$ foram a óbito e $8,3 \%$ abandonaram 0 tratamento. $^{2}$

No Brasil, o Pacto pela Vida prevê o fortalecimento da capacidade de respostas às doenças emergentes $e$ endemias, com ênfase na dengue, hanseníase, malária, influenza e Tb. As metas para o controle da Tb apontam para a necessidade de se atingir pelo menos $85 \%$ de cura dos casos novos de bacilíferos diagnosticados a cada ano. ${ }^{3}$

0 elevado percentual de abandono, o surgimento de formas resistentes do Mycobacterium tuberculosis, a epidemia de AIDS e as desigualdades sociais que restringem o acesso da população a condições dignas de vida, são alguns obstáculos no controle da doença.

Recentemente, a Tb foi classificada como uma doença reemergente nos países desenvolvidos, e manteve sua ocorrência em níveis elevados e eventualmente crescentes nos países em desenvolvimento. A endemia segue como grande problema da saúde pública pela sua capacidade de atingir com maior intensidade as populações marginalizadas, ${ }^{4}$ sendo considerada um marcador social importante em relação às precárias condições de sobrevivência dos indivíduos da maioria das grandes cidades do País. Em geral, estas capitais apresentam $\mathrm{Cl}$ mais elevados, devido à alta densidade demográfica, aliada às situações de exclusão social, o que pode elevar o risco de infecção. Os bolsões de pobreza em certas áreas urbanas evidenciam que estas áreas de exclusão social estão mais propensas ao aparecimento de doenças transmissíveis.

Portanto, no caso de uma doença como a Tb, devem ser considerados outros fatores além da infraestrutura do serviço de saúde e capacitação dos profissionais, como as vulnerabilidades sociais e individuais do doente que podem ser manifestados por falta de residência fixa, desemprego, dificuldade de deslocamento, desconhecimento sobre a doença, entre outros.

Trata-se de um artigo reflexivo, cujo objetivo é suscitar a reflexão sobre as possibilidades de contribuição do referencial da Vigilância da Saúde no controle da Tb.

\section{VIGILÂNCIA DA SAÚDE COMO PROPOSTA DE REORIENTAÇÃO DAS PRÁTICAS NO CONTROLE DA TUBERCULOSE}

0 sistema de saúde brasileiro é palco de disputa entre dois modelos assistenciais que convivem de forma contraditória e ao mesmo tempo complementar: o modelo médico-assistencial privatista e o modelo sanitarista. Alem destes, há esforços de construção de modelos alternativos para impulsionar a saúde, tais como: Cidade Saudável, Saúde da Família, Consórcio Municipal e Intermunicipal, entre outros. ${ }^{5}$

O panorama atual de saúde, com predomínio do modelo médico hegemônico, representado pela baixa efetividade na prestação da atenção à saúde, aponta para a necessidade de, sem negar os modelos anteriores, rever a lógica tradicional que ainda o rege e buscar condições para que, de forma permanente, aproxime-se dos indivíduos, tornando-se mais humanizado e resolutivo. ${ }^{6} \mathrm{~A}$ forma como os serviços de saúde organizam suas práticas influi decisivamente na satisfação dos usuários e no impacto das ações sobre a saúde dos indivíduos.

A proposta da Vigilância da Saúde, contrariamente ao modelo anterior, propõe a incorporação de novos sujeitos, ultrapassando o conjunto de profissionais e trabalhadores de saúde, ao buscar envolver a população organizada. Amplia-se o objeto, pois consideram-se as determinações clínicoepidemiológicas no âmbito individual e coletivo, ao mesmo tempo que contempla a lógica da determinação social do processo saúde-doença.

0 conceito acerca da Vigilância da Saúde surgiu da necessidade de ampliar as ações de Vigilância Epidemiológica, que passou a abrigar, além dos problemas de saúde, as condições de vida da população, focalizando a necessidade de seu monitoramento continuado, deixando de se restringir à identificação e controle de agravos. 0 referencial que orientou a discussão do estudo foi o da Vigilância da Saúde, ${ }^{7}$ entendida como um enfoque que pode contribuir para a atualização das concepções que orientam a reorganização das práticas de saúde. Nesta perspectiva, reforça a dimensão gerencial, caracterizandoa como uma prática que organiza processos de trabalho em saúde sob a forma de operações, para confrontar problemas de 
enfrentamento contínuo, num território delimitado, através de operações montadas segundo os problemas, nos seus diferentes períodos do processo saúde-doença.

Esta concepção é mais abrangente, pois apoia-se na ação intersetorial e procura reorganizar as práticas de saúde no nível local e apresenta as seguintes características básicas: intervenção sobre problemas de saúde (danos, riscos e/ou determinantes); ênfase em problemas que requerem atenção e acompanhamento contínuos; operacionalização do conceito de risco; articulação entre ações promocionais, preventivas e curativas; atuação intersetorial; ações sobre o território e intervenção sob a forma de operações. ${ }^{7}$

A Vigilância à Saúde é uma proposta para a transformação dos determinantes de saúde e doença referenciada pelo conceito de território, participação da população e promoção da saúde, e a reorganização da atenção baseada neste modelo implica novos papéis, novas relações e novas práticas em todos os níveis do sistema. ${ }^{8}$

Nessa perspectiva, a intervenção baseada na Vigilância da Saúde incorpora tecnologias médico-sanitárias, transcendendo o espaço dos serviços de saúde, ou seja, considera 0 território onde vive e trabalha a população, expandindo-se a outros setores e órgãos de ação governamental ou não, envolvendo entidades representativas dos interesses de diversos grupos sociais.

A Vigilância da Saúde possibilita a reorientação da organização do modelo assistencial do Sistema Único de Saúde (SUS), ao incorporar e buscar superar os modelos vigentes, na medida em que propõe a redefinição do objeto, dos meios de trabalho, das atividades, das relações técnicas e sociais, e, também, das organizações de saúde e da cultura sanitária. Assim, "aponta na direção da superação da dicotomia entre as práticas coletivas (vigilância epidemiológica e sanitária) e as práticas individuais (assistência ambulatorial e hospitalar), através das contribuições da nova geografia, do planejamento urbano, da epidemiologia e das ciências sociais em saúde, tendo como suporte político-institucional, o processo de descentralização e de reorganização dos serviços e das práticas de saúde no nível local". ${ }^{9}$

De fato, a Vigilância da Saúde propõe o caminho para repensar o processo de trabalho em saúde, implicando em mudanças não só no processo de trabalho dos profissionais de saúde, mas também nas relações que mantém com outros trabalhadores e, sobretudo, na relação com a população. Desse modo, a intervenção fundamentada na Vigilância da Saúde exige a participação ativa dos profissionais de saúde e da própria população, assim como de trabalhadores de outros setores, além de exigir a reflexão crítica sobre conceitos e as práticas de saúde.

Sabe-se que apenas a disponibilidade de requisitos considerados essenciais, como estrutura física, material permanente e de consumo em quantidade e qualidade, não são suficientes para garantir a qualidade do cuidado prestado.
É necessário ultrapassar barreiras, principalmente aquelas referentes ao campo das relações interpessoais existentes. ${ }^{10}$

As práticas no âmbito da Vigilância da Saúde tem como ponto de partida a noção da territorialização, entendendo o território como o lócus geopolítico onde ocorrem as relações sociais de produção e reprodução social. Assim, o trabalho em saúde deve estar imerso nesse contexto, isto é, pressupõe o reconhecimento e o esquadrinhamento do território, segundo a lógica das relações entre condições de vida, saúde e acesso aos serviços de saúde. 0 propósito fundamental do processo de territorialização é permitir a definição de prioridades em termos de problemas e necessidades dos grupos sociais e, consequentemente, obter impacto positivo sobre os níveis de saúde e condições de vida, através do planejamento e programação local. ${ }^{7}$

A Vigilância da Saúde toma o indivíduo como parte da família, da comunidade, do sistema social e, sobretudo, do território onde se estabelecem as relações de trabalho e de vida. Assim, qualquer intervenção em saúde deve considerar este conjunto de atores e espaço. Nesse contexto, é preciso compreender que melhorar a qualidade de vida e promover a saúde de um indivíduo implica agir no contexto em que ele se insere e no espaço onde vive. ${ }^{11}$

É importante ressaltar que a proposição da Estratégia de Saúde da Família (ESF) está em sintonia com os princípios da Vigilância da Saúde, na medida em que elege como ponto central o estabelecimento de vínculos e a criação de laços de compromisso e corresponsabilidade entre os serviços de saúde e a população.

A ESF, portanto, pode ser considerada como espaço que contribui para a emergência de um novo paradigma no SUS e para o desenvolvimento de novas relações entre os trabalhadores de saúde e a população. No caso específico da Tb, as equipes de saúde da família, por estarem inseridas na comunidade, podem contribuir para o controle da doença, pois o trabalho é realizado de acordo com cada realidade local. Desenvolvem-se atividades de educação em saúde, busca de sintomáticos respiratórios, realiza-se a supervisão medicamentosa, o monitoramento dos contactantes, a busca de faltosos, esclarecendo questões relacionadas à doença, tratamento e possíveis dúvidas que surgem no decorrer do tratamento. 0 foco da Vigilância da Saúde deixa de ser o indivíduo doente e aqueles em risco de adoecer por Tb, passando a incorporar ações intersetoriais, participação da população e atuação no território. Tal fato gera perspectivas de ampliação do acesso aos serviços de saúde e diagnóstico e tratamento dos doentes de Tb. ${ }^{12}$

Uma das estratégias fundamentais para o controle da Tb é a deteç̧ão precoce dos casos, considerando que apenas aguardar que os doentes procurem os serviços de saúde com queixas respiratórias não é suficiente para interromper a cadeia de transmissão da doença. A busca ativa de sintomáticos respiratórios deve ser uma atitude permanente e incorporada à rotina de atividades de todos os membros da equipe de saúde, 
não apenas nos serviços de saúde, mas também nas atividades realizadas na coletividade, seja por meio de visita domiciliar, reuniões com os membros da comunidade, ou outras.

Estudo conduzido em um centro de saúde de Fortaleza, Ceará, evidenciou que o treinamento e a sensibilização dos membros da equipe de saúde da família sobre a temática da $\mathrm{Tb}$ foram capazes de promover um aumento na detecção de casos de Tb na área de estudo. Os autores acreditam que, nos locais onde a ESF está implantada, algumas ações são importantes para o controle da $\mathrm{Tb}$, tais como: treinamento, atualização e estímulo para a equipe em relação à detecção precoce e acompanhamento dos casos e contactantes. ${ }^{13}$

A ESF vem sendo reconhecida como possibilidade na reorientação do modelo de atenção, apesar de, até o momento, não ter viabilizado a ruptura do modelo hegemônico, seja pelo tempo de implantação de pouco mais de uma década, seja por sua cobertura ainda insuficiente para que mostre algum impacto nos indicadores epidemiológicos e na organização da rede de serviços de atenção.

Para que seja possível a operacionalização da Vigilância da Saúde nos territórios de atuação da ESF, diante do controle da Tb, é necessária a superação da lógica assistencial, de caráter individual e curativista, que ainda se faz hegemônica para grande parte das equipes de saúde da família, e, por meio da análise dos problemas identificados, 0 subsídio a formulação de ações estratégicas direcionadas às necessidades dos doentes de Tb e de grupos vulneráveis. ${ }^{9}$

É urgente a necessidade de se criar alternativas para o controle da $\mathrm{Tb}$, principalmente aquelas relacionadas a mudanças de comportamento e a relação entre doentes e profissionais de saúde. Além disso, ressalta-se a importância da existência de um modelo de assistência que priorize uma prática de saúde participativa, que esteja vinculada à comunidade.

Desse modo, o estabelecimento de novas relações entre os agentes de saúde que atuam nas equipes de saúde da família e a população pode promover mudanças na qualidade da atenção e reforçar o compromisso e envolvimento dos sujeitos protagonistas, visto que passam a se sentir "sujeitos ativos", na busca da mudança das condições de saúde e na melhora da qualidade de vida da população.

Isso é viável também considerando-se que os problemas de saúde devem ser enfrentados a partir de ações intersetoriais e não podem ser considerados como responsabilidade exclusiva do setor saúde, visto que a promoção da saúde e a melhoria da qualidade de vida são tidos como pilares fundamentais para o controle da $\mathrm{Tb}$ no Brasil. Para que isto ocorra, faz-se necessária uma integração com outros setores governamentais, no sentido de reduzir a pobreza, a desigualdade e a exclusão social. 0 autor reforça a necessidade de o País buscar alternativas para melhor distribuir a renda, reduzindo o desemprego, além de melhorar o acesso e a qualidade da assistência à saúde, bem como 0 saneamento básico, entre outras prioridades. ${ }^{14}$

\section{APLICABILIDADE DO REFERENCIAL DE VIGILÂNCIA DA SAÚDE EM UM DETERMINADO TERRITÓRIO}

As reflexões do presente artigo foram estimuladas por ocasião de estudo realizado no Município de Ribeirão Preto, que buscou analisar a correlação entre casos novos de $\mathrm{Tb}$ e espaço geográfico, através de técnicas de geoprocessamento. ${ }^{15}$ A estratificação do Município em distintas áreas, segundo condições de vida e a distribuição dos casos da doença, evidenciou que a enfermidade apresentouse irregularmente distribuída no espaço urbano, ressaltando-se que as áreas com maior número de casos corresponderam, em sua maioria, aos bairros mais carentes, o que indica um grau de relação entre as condições de vida dos indivíduos que residem nas áreas mais afetadas ou com aglomerações caracterizadas por condições socioeconômicas precárias.

A Vigilância da Saúde reconhece o território como conceito fundamental para viabilizar a reorientação do processo de trabalho e é nesse espaço onde se busca estabelecer a definição de problemas e prioridades, assim como os meios para atender as necessidades de saúde da coletividade. Acredita-se que a Vigilância da Saúde deve estar apoiada em três pilares fundamentais: território, problemas e práticas de saúde. ${ }^{6}$ Nesse caso, o território é considerado o lócus privilegiado de atuação, onde podem ser definidos problemas e identificadas as necessidades de saúde.

A identificação das áreas consideradas de risco e de grupos com diferentes vulnerabilidades à Tb é fundamental para a orientação de ações de saúde com base na Vigilância da Saúde, pois permite um planejamento focalizado a estes grupos, em função da necessidade de melhorias sociais e de saúde. Este referencial teórico permite compreender que as ações de controle da Tb não podem continuar voltadas apenas à identificação e tratamento dos doentes, pois apenas estas estratégias não são suficientes para modificar a situação epidemiológica da doença. Os esforços devem estar voltados ao contexto de vida em que os doentes estão inseridos.

$\mathrm{Na}$ perspectiva da Vigilância da Saúde, o conhecimento da distribuição da doença no espaço pode potencializar a necessidade da prioridade de ações de saúde, ressaltando-se que a identificação de áreas heterogêneas permite definir grupos prioritários para a intervenção, estabelecimento de ações conjuntas com outros setores e equipamentos sociais para atender as necessidades dos doentes, que muitas vezes extrapolam o âmbito da saúde, mas são determinantes do processo de saúde-doença vivenciado. 


\section{CONCLUSÃO}

Este estudo evidenciou que o conceito de Vigilância da Saúde pode trazer contribuições para o controle de uma doença de distribuição marcadamente heterogênea como a Tb, através do conhecimento da distribuição da doença no espaço urbano, 0 que torna possível a identificação de grupos prioritários que necessitam de intervenções mais específicas. Ressalta-se a necessidade de ampliar a visão de mundo dos profissionais de saúde tanto sobre os processos saúde-doença quanto sobre os processos de trabalho e produção em saúde para qualificar as ações voltadas ao controle da Tb, por meio de um novo olhar sobre o objeto de intervenção e criação de novas tecnologias para intervir nesses processos.

\section{REFERÊNCIAS}

1-Monroe AA, Cardoso-Gonzáles RI, Palha PF, Sassaki CM, Ruffino-Netto A, Vendramini $S H F$, et al. Envolvimento de equipes da atenção básica à saúde no controle da tuberculose. Rev Esc Enferm USP. 2008 jun; 42(2): 26267.

2-World Health Organization-WHO. Global tuberculosis control: surveillance, planing, financing. Geneva: WHO Report; 2009.

3- Ministério da Saúde(BR). Diretrizes operacionais dos pactos pela vida, em defesa do SUS e de gestão. Brasília(DF); 2006.

4- Santos J. Resposta brasileira ao controle da tuberculose. Rev Saude Publica. 2007 set; 41 (supl 1): 89-93.

5- Paim JS. Modelos de atenção e vigilância da saúde. In: Almeida-Filho N, Rouquayrol MZ, organizadores. Epidemiologia\&Saúde. Rio de Janeiro: Medsi; 2003. p.567-86.

6- Oliveira CM, Casanova A0. Vigilância da saúde no espaço de práticas da atenção básica. Cienc Saude Colet. 2009 maio; 14(3): 929-36.

7- Teixeira CF, Paim JS, Vilasbôas AL. SUS, modelos assistenciais e vigilância da saúde. Inf Epidemiol SUS. 1998 abr/jun; 7(2): 7-28.

8- Rodrigues VM, Fracolli LA, Oliveira MAC. Possibilidades e limites do trabalho de vigilância epidemiológica em direção à vigilância á saúde. Rev Esc Enferm USP. 2001 dez; 35(4): 313-19.

9- Teixeira CF.Promoção e vigilância da saúde no contexto da regionalização da assistência à saúde no SUS. Cad Saude Publica. 2002; 18(supl I):15362.

10- Freire MAM, Morais FT, Albuquerque BMD, Melo ECP. 0 processo de trabalho em uma unidade básica no Município do Rio de Janeiro. Esc Anna Nery. 2008 set; 12(3): 508-14.

11- Campos CEA. 0 desafio da integralidade segundo as perspectivas da vigilância da saúde e da saúde da família. Cienc Saude Colet. 2003; 8(2): 569-84.
12- Souza JN, Bertolozzi MR. A vulnerabilidade à tuberculose em trabalhadores de enfermagem em um hospital universitário. Rev Latinoam Enfermagem. 2007 abr; 15(2): 259-66.

13- Façanha MC, Melo MA, Vasconcelos FF, Sousa JRP, Pinheiro AS, Porto IA et al. Treinamento da equipe de saúde e busca ativa na comunidade: estratégias para a detecção de casos de tuberculose. J Bras Pneumol. 2009 maio; 35(5): 449-54.

14- Guimarães MJB, Marques NM, Melo Filho DA, Szwarcwald CL. Condição de vida e mortalidade infantil: diferenciais intra-urbanos no Recife, Pernambuco, Brasil. Cad Saude Publica. 2003 set; 19(5):1413-24.

15- Hino P. Padrões espaciais da tuberculose associados a um indicador adaptado de condição de vida no município de Ribeirão Preto[tese]. Ribeirão Preto: Escola de Enfermagem, Universidade de São Paulo; 2008. 